\title{
Impact of stimulus format and reward value on quantity discrimination in capuchin and squirrel monkeys
}

\author{
Regina Paxton Gazes ${ }^{1}$ • Alison R. Billas ${ }^{2}$ - Vanessa Schmitt ${ }^{3,4,5}$
}

Published online: 24 August 2017

(C) Psychonomic Society, Inc. 2017

\begin{abstract}
Quantity discrimination abilities are seen in a diverse range of species with similarities in performance patterns, suggesting common underlying cognitive mechanisms. However, methodological factors that impact performance make it difficult to draw broad phylogenetic comparisons of numerical cognition across studies. For example, some Old World monkeys selected a higher quantity stimulus more frequently when choosing between inedible (pebbles) than edible (food) stimuli. In Experiment 1 we presented brown capuchin (Cebus [Sapajus] paella) and squirrel monkeys (Saimiri sciureus) with the same two-choice quantity discrimination task in three different stimulus conditions: edible, inedible, and edible replaced (in which choice stimuli were food items that stood in for the same quantity of food items that were given as a reward). Unlike Old World monkeys, capuchins selected the higher quantity stimulus more in the edible condition and squirrel monkeys showed generally poor performance across all stimulus types. Performance patterns
\end{abstract}

Electronic supplementary material The online version of this article (doi:10.3758/s13420-017-0295-9) contains supplementary material, which is available to authorized users.

Regina Paxton Gazes

reggie.gazes@bucknell.edu

1 Department of Psychology and Program in Animal Behavior, Bucknell University, Lewisburg, PA, USA

2 Program in Animal Behavior, Bucknell University, Lewisburg, PA, USA

3 Zoo Heidelberg, Heidelberg, Germany

4 Centre for Organismal Studies, University of Heidelberg, Heidelberg, Germany

5 Zoo Atlanta, Atlanta, GA, USA suggested that differences in subjective reward value might motivate differences in choice behavior between and within species. In Experiment 2 we manipulated the subjective reinforcement value of the reward by varying reward type and delay to reinforcement and found that delay to reinforcement had no impact on choice behavior, while increasing the value of the reward significantly improved performance by both species. The results of this study indicate that species presented with identical tasks may respond differently to methodological factors such as stimulus and reward types, resulting in significant differences in choice behavior that may lead to spurious suggestions of species differences in cognitive abilities.

Keywords Numerical processing $\cdot$ Motivation $\cdot$ Subjective reinforcement $\cdot$ Delay to reinforcement

The ability to discriminate between quantities has been shown by a diverse range of species including primates, birds, dolphins, fish, rodents, and canids (nonhuman primates: Addessi, Crescimbene, \& Visalberghi, 2008; Beran, 2001; Jordan \& Brannon, 2006; dolphins: Kilian, von Fersen, \& Gunturkun, 2003; birds: Kelly, 2016; fish: Agrillo, Dadda, \& Bisazza, 2007; Agrillo, Dadda, Serena, \& Bisazza, 2008; rodents: Panteleeva, Reznikova, \& Vygonyailova, 2013; canids: Baker, Shivik, \& Jordan, 2011; Utrata, Viranyi, \& Range, 2012). Many show similar performance patterns that may indicate common underlying cognitive mechanisms. For example, when choosing the larger quantity stimulus from two choices, many species show a ratio effect consistent with Weber's law, showing increased response latency and decreased accuracy with increasing ratio between the two comparator quantities (e.g., 2 vs. 3 has a ratio of $0.67,4$ vs. 5 has a ratio of 0.80 ), and a distance effect, showing increased accuracy and decreased response 
latency with increasing distance between the two comparator quantities (e.g. 2 vs. 3 has a distance of 1, 2 vs. 5 has a distance of 3; baboons: Barnard et al., 2013; humans: Cantlon \& Brannon, 2006; capuchin monkeys: Evans, Beran, Harris, \& Rice, 2009; fish: Gomez-Laplaza \& Gerlai, 2016; apes: Hanus \& Call, 2007; rhesus monkeys: Jordan \& Brannon, 2006; jays: Kelly, 2016; lemurs: Merritt, Maclean, Crawford, \& Brannon, 2011; pigeons: Roberts, 2005; but see wolves: Utrata et al., 2012). These consistencies in performance suggest a shared approximate representation of quantity across species (Beran, 2008; Cantlon, 2012).

Despite similarities in quantity discrimination performance patterns, differences in success across species may indicate informative species differences in numerical competence (Agrillo \& Bisazza, 2014). For example, in contrast to orangutans, gorillas performing a quantity discrimination task require more extensive training to reach above-chance performance and perform less accurately overall (Anderson, Stoinski, Bloomsmith, \& Maple, 2007; Anderson et al., 2005). This may suggest that gorillas and orangutans differ fundamentally in the cognitive mechanisms they employ to discriminate quantities. However, quantity discrimination tasks require integration of many fundamental cognitive processes to produce the final behavioral output. For example, animals must be motivated to perform the task, attend to the stimuli, inhibit tendencies to reach for the first choice they see, and accurately represent the choice stimuli as quantities before making the critical task comparison. Differences in choice behavior are therefore not limited to differences in quantity discrimination ability but may be explained by differences at any point in the processing chain. For example, gorillas and orangutans may employ the same cognitive processes when comparing quantities, but gorillas could be less motivated to obtain the food rewards, resulting in the observed poor performance. Dissociating the contributions of basic cognitive processes to performance on these tasks is therefore essential for making broader phylogenetic comparisons of numerical cognition (Agrillo \& Bisazza, 2014).

To determine how basic cognitive processes impact quantity discrimination abilities in Old World monkeys, Schmitt and Fischer (2011) studied the impact of stimulus format and reward contingency on choice behavior in olive baboons (Papio anubis) and long-tailed macaques (Macaca fascicularis). Monkeys chose between two quantities of items and received a food reward corresponding to the number of stimuli in the option they selected. Stimuli were presented in three conditions: edible, in which the choice stimuli were the food items used as a reward; inedible, in which the choice stimuli were inedible pebbles that stood in for the same quantity of food items that were given as a reward; and edible replaced, in which choice stimuli were food items that stood in for the same quantity of food items that were given as a reward. Each condition was preceded by a short training phase to assure that monkeys were familiar with the choice and reward contingencies in that condition. The Old World monkeys chose the higher quantity stimulus less frequently in the edible condition than in the inedible and edible replaced conditions (Schmitt \& Fischer, 2011). Despite significant performance differences across stimulus types, consistent abovechance performance, ratio, and distance effects suggest that animals were relying on the same approximate magnitude system when making their choices across all conditions. Higher accuracy with inedible than edible stimuli may therefore be driven by differences in basic processing of the choice items. For example, the visual presence of food in the edible conditions may make it more likely that a monkey will make the first available response, rather than taking time to compare the choices and make the response that would maximize their rewards (Schmitt \& Fischer, 2011). However this lack of inhibitory control (Brucks, Soliani, Range, \& Marshall-Pescini, 2017) alone could not explain the results, as it would predict poor performance in the edible replaced condition, where food items were also used as choice stimuli. The authors suggest that the best explanation for the differences in choice behavior across the three stimulus conditions by Old World monkeys may be that they are unable to represent the food items in the edible condition as both the choice stimuli and the reward. Difficulty with this "dual representation" of food items would drive down performance in the edible condition but would not impact choice behavior in the inedible or edible replaced conditions in which there were distinct choice and reward items (Schmitt \& Fischer, 2011). As different stimulus processing mechanisms predict distinct performance patterns across conditions, stimulus manipulations in quantity discrimination tasks may be particularly informative for determining the role of basic cognitive processes in choice behavior.

Data from capuchin monkeys suggest that there may be some qualitative difference in how New and Old World primates process edible and inedible stimuli in quantity discrimination tasks. Capuchin monkeys, a New World primate species, presented with edible and inedible choice stimuli show the opposite effect to that shown by baboons and macaques, showing lower accuracy on quantity discrimination, size discrimination, and delayed gratification tasks with inedible items than with edible items (Addessi et al., 2008; Evans, Beran, Paglieri, \& Addessi, 2012; Truppa, Carducci, Trapanese, \& Hanus, 2015). However, capuchin monkeys are the only species of New World primate that has been systematically tested with both edible and inedible stimuli, and due to differences in methodologies across studies it is not possible to draw comparative conclusions about the impact of stimulus type on choice behavior. In Experiment 1, we applied the same methods as those presented in Schmitt and Fischer (2011) to capuchin and squirrel monkeys, expanding the species tested and allowing for direct comparisons of the impact of stimulus format on quantity 
discrimination across these primate groups. To further disentangle possible factors influencing choice in the quantity discrimination task, in Experiment 2 we manipulated the value of the reward to assess how motivation differences influenced choice behavior between and within species.

\section{Experiment 1}

Both capuchin and squirrel monkeys can discriminate quantities. Squirrel monkeys chose the larger quantity of food in a two-choice task (McKenzie, Cherman, Bird, Naqshbandi, \& Roberts, 2004), the smaller quantity of food in a reversereward contingency task (Anderson, Awazu, \& Fujita, 2000, 2004), can learn relative quantity discriminations with inedible choice stimuli (Terrell \& Thomas, 1990; Thomas \& Chase, 1980; Thomas, Fowlkes, \& Vickery, 1980), and learn to select the larger Arabic numeral when those numerals are associated with the corresponding quantity of food (Olthof, Iden, \& Roberts, 1997). Capuchin monkeys select the larger quantity of items when presented with edible (Addessi et al., 2008; Beran, Evans, Leighty, Harris, \& Rice, 2008b) and inedible choice stimuli (Addessi et al., 2008), and individuals trained to order quantities 1-4 spontaneously transfer to quantities 5-9 (Judge, Evans, \& Vyas, 2005). That both species can discriminate quantities with edible and inedible stimuli indicates they are promising New World primate species in which to study the impact of stimulus type on quantity discrimination.

\section{Method}

Subjects and housing Subjects were seven brown capuchin monkeys (Cebus [Sapajus] apella; five females; mean age $8.43 \pm 3.51$ years) and six squirrel monkeys (Saimiri sciureus; six females; mean age $10.17 \pm 3.49$ years) housed in social colonies at Bucknell University. All subjects had extensive experience with manual cognitive testing (e.g., Judge \& Bruno, 2012; Judge, Evans, Schroepfer, \& Gross, 2011; Kurdziel \& Judge, 2007; Marsh, Vining, Levendoski, \& Judge, 2015; Zander \& Judge, 2015) but not with quantity discrimination specifically. All subjects were tested alone in their home cage by temporarily closing off small sections of the housing area. Animals maintained visual and auditory contact with their social group during testing. Food and water were available ad libitum.

Apparatus Choices were presented on top of a flat table-like surface approximately $0.86 \mathrm{~m} \times 0.35 \mathrm{~m} \times 1.42 \mathrm{~m}$ that allowed monkeys to sit on a perch in their home cage and interact with the choices. Each trial presented two choice quantities of stimuli presented on opaque plastic plates $(13.97 \mathrm{~cm} \times 13.97 \mathrm{~cm})$ that covered opaque Tupperware containers $(13.97 \mathrm{~cm} \times$ $13.97 \mathrm{~cm} \times 6.4 \mathrm{~cm}$ deep). For the inedible and edible replaced conditions the food rewards were placed out of sight under the opaque plastic plates that held the choice stimuli. Rewards (and choice stimuli for edible and edible replaced conditions) consisted of one-eighth piece of a grape. All quantities of food reinforcers and choice stimuli therefore refer to one eighth of a grape. Choice stimuli for the inedible condition were black aquarium marbles.

\section{Procedure}

General procedure All procedures were approved by the Bucknell IACUC. Bucknell University's research facility is accredited by the USDA.

Each trial began with an opaque occluder placed between the subject and the choice containers to block the view of the trial setup. Once stimuli and rewards were in place, the occluder was raised and the subject was prompted to make their choice by the vocal cue "Which one [name of subject]." During this prompt, the experimenter placed her hands at her sides and stared at the center of the choice tray with her head tilted down to avoid cueing the subject toward either choice. Subjects indicated their choice by pointing to or reaching for one of the two choice quantities. Subjects received the number of food rewards corresponding to the number of choice stimuli they selected, regardless of whether they chose the smaller or larger quantity. Chosen food rewards were placed into the experimenter's open palm and then offered to the subject. Subjects could only choose one option per trial. The location of the larger quantity stimulus varied semirandomly across trials. All training and testing sessions consisted of 10 trials. Subjects received one session per day, 5 days per week. Trials were video recorded.

Conditions Subjects received three different testing conditions - edible, inedible, and edible replaced - that varied in the type of stimuli used to represent the quantities (see Fig. 1). Each condition consisted of both a training and a testing phase. The three conditions were presented sequentially with the order of presentation counterbalanced across subjects.

Edible condition. A quantity of grape pieces was presented on each of the two choice plates. After pointing to one of the two choices, the stimulus grapes from the top of the plate were placed into the experimenter's open palm and offered to the monkey.

Inedible condition. A quantity of round, black, uniformly sized aquarium marbles was presented on each of the two choice plates. After monkeys made their choice by pointing to one of the two choices, the plate holding the marbles for that choice was removed, and the monkey received that quantity of grapes held underneath. These 

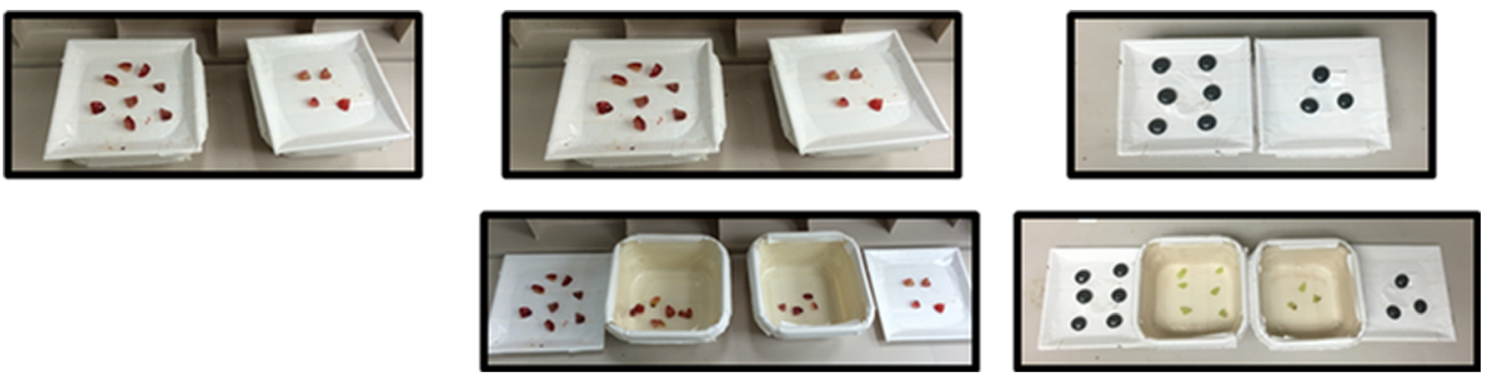

Fig. 1 Experimental set up for (left) edible, (center) edible replaced, and (right) inedible conditions. Images on the top show stimuli visible to the monkeys at the time of choice. Choice stimuli for edible and edible replaced conditions were one-eighth pieces of grapes; choice stimuli for

rewards were placed into the experimenter's open palm and presented to the subject. Monkeys could not see the food reward at the time of their choice.

Edible replaced condition. At choice this condition looked identical to the edible condition: monkeys chose between two quantities of grapes presented on the choice plates. However, they did not receive the grapes on the plate they chose. Instead, as in the inedible condition, the choice plate was removed and monkeys received an identical quantity of grapes hidden in the container under the plate. Rewards were placed into the experimenter's open palm and presented to the subject.

Training Each of the three conditions began with a training phase. These training phases exposed the subjects to the stimulus contingency for that condition. Subjects received 10 trial sessions containing quantity pairs 2 versus 7 and 1 versus 6 semirandomly intermixed (five trials of each quantity pair) until they chose the larger quantity on eight of 10 trials (80\% accuracy) for one session. After reaching this criterion, subjects moved on to the testing phase for that condition.

Testing Testing differed from training in that primates were presented with 20 novel quantity pair combinations of quantities 1 through 8 (see Table 1). Each individual received four 10-trial sessions per condition, with each of the 20 possible quantity pairs presented semirandomly twice over the course of the four sessions, resulting in a total of 40 test trials. Pairs differed in the numerical distance between the two quantities and in the numerical ratio between the two quantities (see Table 1).

Data analysis A semirandom subset of videotaped trials across conditions and subjects were scored to determine the delay between choice and receiving a reward. A repeatedmeasures ANOVA compared this delay to reinforcement across the three conditions (edible, edible replaced, inedible).

Correct choices were scored as the choice of the higher quantity item in the pair. Analyses focused on pairs with a the inedible condition were round aquarium marbles. Images on the bottom show reward stimuli for the edible replaced and inedible conditions after the plates containing the choice stimuli has been removed

distance greater than zero. One-sample $t$ tests compared performance to $50 \%$ chance level. To account for both repeated sampling of individual subjects across three conditions and the comparison between species, linear mixed-effect models, with subject as a random factor, were conducted (Boisgontier \& Cheval, 2016; Bolker et al., 2009). Linear mixed-effect models confer an advantage over traditional ANOVAs for analyzing repeated-measures data in that they use all available data for each subject, do not require assumptions of sphericity, are unaffected by missing data, and can account for variation between individuals (random effects; Gueorguieva \& Krystal, 2004). The model analyzing choice behavior during test trials across the three conditions included main effects of condition order (first, second, third), the natural log of the ratio between quantities in a pair, and the distance between the quantities in a pair on performance. If subjects showed an effect of ratio and distance, it would suggest that they were attending to the relative quantities of the items in the pairs when making their choices. The model additionally included main effects of species (capuchin, squirrel monkey), condition (edible, inedible, and edible replaced), and the interaction between these variables (species $\times$ condition) on choice behavior. A significant interaction between species and condition would indicate differences in performance patterns across the conditions by each species, suggesting differences in processing of the stimulus types. Models were fitted using software R Version 3.1.2 ( $\mathrm{R}$ Core Team, 2014) and R package lmerTest (Kuznetsova, Brockhoff, \& Christensen, 2015). Satterthwaite

Table 1 Pairs of numerical quantities presented in the test phases

\begin{tabular}{lllll}
\hline 0 & 1 & 2 & 3 & 4 \\
\hline $1: 1(1.00)$ & $2: 1(0.5)$ & $3: 1(0.33)$ & $4: 1(0.25)$ & $5: 1(0.20)$ \\
$2: 2(1.00)$ & $3: 2(0.67)$ & $4: 2(0.50)$ & $5: 2(0.40)$ & $6: 2(0.33)$ \\
$3: 3(1.00)$ & $4: 3(0.75)$ & $5: 3(0.60)$ & $6: 3(0.50)$ & $7: 3(0.43)$ \\
$4: 4(1.00)$ & $5: 4(0.80)$ & $6: 4(0.67)$ & $7: 4(0.57)$ & $8: 4(0.50)$ \\
\hline
\end{tabular}

Note. Columns indicate distance between items. Ratios between the test items in each pair indicated in parentheses. Distance 0 pairs were added as a side bias check in case of chance performance 
approximations were used to approximate significance levels and degrees of freedom. Degrees of freedom for linear mixedeffect models are higher than traditional ANOVAs because linear mixed-effect models take every trial into account, rather than focusing on subject averages. For all group difference parameter estimates, 95\% confidence intervals are presented as standardized measures of effect sizes, and slopes and standard errors are presented for all continuous variables. All tests used an alpha level of $p=.05$, except in cases with multiple comparisons, where a more conservative value of $p=.01$ was used. Mean choice of the higher quantity stimulus and standard deviations for each condition are presented in text and presented for each test pair across all conditions in the Supplemental Materials (Tables S1 and S2).

\section{Results}

Training There were no differences in the number of training sessions required to reach criterion between conditions or species $(M=2.51 \pm 1.81)$, linear mixed-effect model, condition $\times$ species; main effect of condition, $F(2,22)=0.368, p=.696$; main effect of species, $F(1,11)=0.001, p=.974,95 \% \mathrm{CI}$ $[-1.48,2.38]$; species $\times$ condition interaction, $F(2,22)=$ $2.382, p=.116$.

Testing Using a conservative $p$ value of .01 to correct for multiple comparisons, one-sample $t$ tests revealed that both species selected the higher quantity item significantly above chance on test trials in all conditions, except for the squirrel monkeys in the edible condition, where they were trending toward above-chance performance, $t(5)=3.94, p=.011$ (see Table 2 and Fig. 2). A linear mixed-effect model revealed significant main effects of ratio and distance consistent with Weber's law and the distance effect; choice of the higher quantity stimulus increased as the ratio between the stimuli decreased (slope $=-0.10, S E=0.04), F(1,1244)=-5.47, p=$ $.020,95 \%$ CI $[0.02,0.18]$, and distance between the stimuli increased (slope $=0.03, S E=0.01), F(1,1244)=5.26, p=$ $.022,95 \%$ CI $[0.01,0.06]$. As in Old World monkeys, these patterns suggest that monkeys were comparing the quantities of the stimuli when making choices, and provide further evidence for an approximate magnitude representation underlying quantity discrimination across primates (Barnard et al., 2013; Beran, 2008; Cantlon \& Brannon, 2006; Cantlon, 2012).

The linear mixed-effect model also showed no main effect of condition order, $F(1,1246)=0.02, p=.888,95 \% \mathrm{CI}$ $[-0.03,0.03]$, on choice behavior. There was no main effect of condition, $F(2,1246)=2.36, p=.095$, but a significant main effect of species, $F(2,11)=12.90, p<.004,95 \%$ CI $[-0.33,-0.14]$, and a significant interaction between species and condition, $F(2,1245)=5.51, p<.004$. The significant effect of species indicates that overall, capuchins choose the larger quantity more frequently than did squirrel monkeys. The significant interaction between species and condition indicates differences in choice patterns across the three stimulus conditions by capuchins and squirrel monkeys (see Fig. 2). Two follow-up linear mixed models (one per species) with subject as a random factor and condition as a fixed factor were used to clarify the species $\times$ condition interaction. Models revealed that the significant interaction between species and condition was driven by high levels of choice of the higher quantity stimulus by capuchin monkeys in the edible condition (contrasts on main effect of condition): edible vs. edible replaced, $t(663)=-2.57, p=.01,95 \%$ CI $[-0.17,-0.02]$; edible vs. inedible, $t(663)=-3.97, p<.001,95 \%$ CI $[-0.23$, $-0.08]$. Choice behavior by capuchins did not differ between the inedible and edible replaced conditions, $t(663)=1.40, p=$ $.161,95 \%$ CI $[-0.02,0.13]$, and squirrel monkeys showed no differences in choice behavior across the three conditions (contrasts on main effect of condition, all $p \mathrm{~s}>.10$ ).

A repeated-measures ANOVA on the delay between choice and receiving reinforcement across the three conditions revealed that delay to reinforcement was shortest in the edible condition, followed by the edible replaced condition, with the inedible condition showing the longest delay $\left(M_{\text {Edible }}=2.42 \mathrm{~s} \pm\right.$ $\left.1.10 ; M_{\text {EdibleReplaced }}=3.91 \mathrm{~s} \pm 0.81 ; M_{\text {Inedible }}=5.33 \mathrm{~s} \pm 0.85\right)$, main effect of condition, $F(2,27)=25.50, p<.001, \eta_{\mathrm{p}}{ }^{2}=0.65$. This was due to the added time in the edible replaced and inedible conditions to remove the plate holding the choice stimuli and lift the food rewards from inside the container.

\section{Discussion}

Performance patterns across the three stimulus conditions by capuchin and squirrel monkeys differed from those of Old World monkeys tested using the same methods (Schmitt \& Fischer, 2011). Baboons and macaques chose the higher quantity stimulus significantly less in the edible condition than in the other conditions. Consistent with previous literature (Addessi et al., 2008), capuchin monkeys showed the opposite pattern, selecting the higher quantity stimulus more frequently in the edible condition than in either of the other two conditions. Squirrel monkeys showed no differences in choice behavior across the three conditions. That, like Old World monkeys, New World monkeys overall showed above-chance performance, ratio, and distance effects suggests that all species were attending to the relative quantity of the stimuli when making their choices and were likely relying on similar approximate magnitude representations to make their choices. Differences in performance patterns across the species are therefore likely driven by differences in basic processing of the choice stimuli.

While performance patterns by Old World monkeys may be best explained by difficulty representing edible stimuli as 
Table 2 Means (proportions), standard deviations, results of one-sample $t$ tests, and 95\% confidence intervals on proportion choice of higher quantity stimulus for capuchin and squirrel monkeys in Experiment 1

\begin{tabular}{llll}
\hline Species & Edible & Edible replaced & Inedible \\
\hline Capuchins & $M=0.87 \pm .34$ & $M=0.77 \pm .42$ & $M=0.72 \pm .46$ \\
& $t(6)=13.84, p<.001$ & $t(6)=8.14, p<.001$ & $t(6)=4.70, p=.003$ \\
& $95 \%$ CI $[0.31,0.44]$ & $95 \%$ CI $[0.20,0.36]$ & $95 \% \mathrm{CI}[0.11,0.34]$ \\
Squirrel monkeys & $M=0.64 \pm .48$ & $M=0.70 \pm .46$ & $M=0.66 \pm .47$ \\
& $t(5)=3.94, p=.011$ & $t(5)=4.49, p=.006$ & $t(5)=4.44, p=.007$ \\
& $95 \%$ CI $[0.05,0.23]$ & $95 \%$ CI $[0.09,0.31]$ & $95 \% \mathrm{CI}[0.07,0.25]$ \\
\hline
\end{tabular}

Note. Results presented in italics indicate above-chance performance using $p=.01$

both choice and reward (Schmitt \& Fischer, 2011), this mechanism cannot account for the performance patterns shown by New World monkeys. One of the most salient differences between the edible and inedible stimuli is the visual access to food reward. This obvious presence of food could result in two opposite performance patterns for edible stimuli. First, the presence of edible stimuli could result in low inhibitory control that leads to decreased accuracy (Boysen, Berntson, Hannan, \& Cacioppo, 1996). Inhibitory control is the ability to inhibit an immediate action in favor of a more advantageous behavior (Brucks et al., 2017). Monkeys with poor inhibitory control would be more likely to respond to the first choice they see on a quantity discrimination task rather than taking the time to make an accurate discrimination and select the higher quantity stimulus. Inhibitory control may be particularly difficult when presented with visible edible stimuli; indeed, apes and capuchin monkeys perform worse on reverse-reward contingency tasks with visible food rewards than when food is covered (Vlamings, Uher, \& Call, 2006) or represented by tokens (e.g., Arabic numerals or "low value" and "high value" tokens; Addessi \& Rossi, 2011; Boysen et al., 1996). Differences in inhibitory control between species could

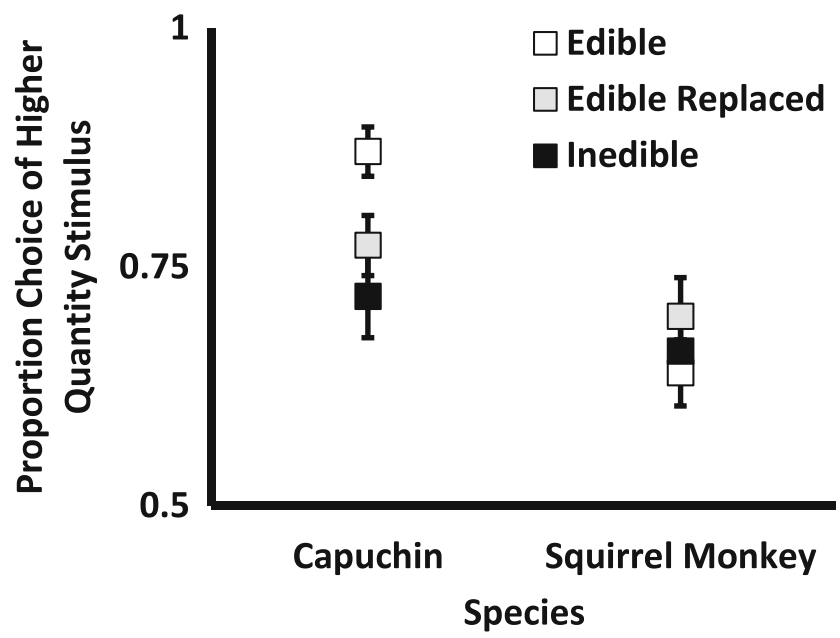

Fig. 2 Proportion choice of higher quantity stimulus across the three conditions (edible, edible replaced, and inedible) for capuchins and squirrel monkeys. Error bars represent standard error of the mean therefore lead to differences in performance on quantitydiscrimination tasks, such that those with poor control would perform worse on discriminations with visible edible stimuli than with inedible stimuli. However, capuchin monkeys in the present experiment chose the higher quantity stimulus most frequently in the edible condition, which would require the highest levels of inhibitory control. Inhibitory control alone is therefore not sufficient to explain stimulus-specific performance patterns by capuchin monkeys. As squirrel monkeys showed equally low rates of choice of the higher quantity item across all stimulus conditions, it is possible that they exhibit generally low inhibitory control, selecting whichever choice is most readily available. However, squirrel monkeys have been shown to systematically select a lower quantity of preferred food over a higher quantity of less preferred food, suggesting that they can inhibit their tendency to reach for a larger quantity in favor of a more desired reward (McKenzie et al., 2004).

Second, visual access to food reward at choice could result in increased motivation to obtain rewards. Importantly, on quantity-discrimination tasks such as those presented in Experiment 1, there is no "incorrect" response - animals are reinforced regardless of their choice. Selection of the higher quantity stimulus therefore must be driven not just by a drive to obtain rewards but by a drive to obtain the most rewards. Quantity discrimination tasks may therefore be particularly susceptible to the influence of motivation. Monkeys perform better and faster on cognitive tasks when they are more highly motivated (Gossette \& Feldman, 1968; Minamimoto, La Camera, \& Richmond, 2009; Schrier \& Harlow, 1956; Schubiger, Kissling, \& Burkart, 2016). Motivation levels are related to the subjective value of the reward, which can be based on a combination of objective features, such as reinforcement rates (Schubiger et al., 2016) or the quantity and visual availability of food (Minamimoto et al., 2009; Schrier \& Harlow, 1956; Veling \& Bijleveld, 2015), and subjective features, such as internal drive state or preference for food type (Gossette \& Feldman, 1968; McKenzie et al., 2004; Minamimoto et al., 2009). Visual access to food rewards in the case of edible choice stimuli may therefore increase the subjective value of the reward, resulting in increased 
motivation to select the higher quantity stimulus. However, if the visual presence of food motivated capuchin monkeys to select the higher quantity item more frequently in the edible condition, they should have shown similar performance patterns in the edible replaced condition, where food was also visible at choice. Capuchins chose the higher quantity item significantly less frequently in the edible replaced condition, suggesting the visual access to reward alone is not sufficient to explain their choice behavior. However, the edible condition differed from the inedible condition in one other important aspect that may have resulted in higher subjective reward values; the delay to reinforcement was significantly shorter in the edible condition than in the inedible condition. This is consistent with previous studies in which edible stimuli are often associated with a shorter delay to reward than are inedible stimuli. For example, edible stimuli are generally presented directly to the subject after choice, while inedible stimuli, such as tokens, often must be traded back to the experimenter to receive the reward (Addessi et al., 2008; Evans et al., 2012). Importantly, decreasing the delay to receive the reward increases the subjective value of a reinforcer (see Mazur, 2001, for review; Minamimoto et al., 2009). The relatively short delay to reinforcement in the edible condition may therefore have increased the subjective value of the reward, resulting in the choice patterns shown by capuchin monkeys.

Capuchins may have been more motivated to obtain rewards in the edible condition due to higher subjective reward value from either the visual availability of food items, the relatively short delay to reinforcement, or a combination of both. The generally low selection of the higher quantity item by squirrel monkeys may be explained by poor inhibitory control, although previous research suggest that in certain tasks squirrel monkeys can exercise control (McKenzie et al., 2004). While many studies have shown that capuchin monkeys can consistently discriminate quantities (Addessi et al., 2008; Beran et al., 2008a, b; Evans et al., 2009; Judge et al., 2005), the results of quantity discrimination studies with squirrel monkeys have been mixed (Adachi, Anderson, \& Fujita, 2011; McKenzie et al., 2004; Thomas \& Chase, 1980; Thomas et al., 1980). It is therefore possible that capuchin monkeys are qualitatively better at discriminating quantities than are squirrel monkeys, and that the present quantitycomparison task approached the limits of quantitydiscrimination ability by squirrel monkeys. Alternatively, while grapes were rewarding enough to support learning and regular participation in the study by squirrel monkeys, squirrel monkeys may have assigned a lower subjective value to these rewards than did capuchin monkeys. Like capuchins in the inedible condition, squirrel monkeys may have been poorly motivated to obtain the maximum number of rewards in all conditions, resulting in lower rates of selection of the higher quantity stimulus. Previous research partially supports this hypothesis - two squirrel monkeys choosing between quantities of food items performed at chance when choosing between nonpreferred food items, but performance by one of the subjects increased significantly when choosing between preferred food items (McKenzie et al., 2004; Olthof et al., 1997).

In Experiment 2 we introduced two subjective motivation manipulations - changing the delay to reinforcement and offering preferred food items as rewards - to determine whether the within and between species performance differences observed in Experiment 1 could be explained by differences in motivation to obtain rewards.

\section{Experiment 2}

The results of Experiment 1 suggested that motivation to obtain food rewards may have played an important role in quantity-discrimination performance by capuchins and squirrel monkeys. Squirrel monkeys may have been generally unmotivated to obtain rewards due to low subjective value of the reinforcers, resulting in the observed low rates of selection of the higher quantity stimuli across all conditions. Capuchin monkeys may have been more motivated to obtain food rewards in the edible condition, due to a combination of the visual availability of the food reinforcer and the relatively shorter delay to reinforcement.

In Experiment 2 we manipulated motivation by changing the subjective value of the rewards for edible and inedible stimuli in four new conditions. First, we presented edible and inedible conditions identical to Experiment 1, but instead of grapes as reinforcers, monkeys were presented with their preferred food item. If performance by squirrel monkeys in Experiment 1 was due to low subjective value of the grape rewards, then presenting a preferred food item as reinforcement should increase selection of the higher quantity stimulus in both edible and inedible conditions. However if performance by squirrel monkeys was due to generally poor quantity discrimination ability or poor inhibitory control, then changing the reinforcement should not dramatically impact choice behavior. If choice of the higher quantity stimulus by capuchin monkeys in the edible condition was driven by increased motivation due to a higher subjective reward value for visually available food items, then presenting a preferred food item as a reinforcer should likewise increase motivation to obtain rewards, resulting in increased choice of the higher quantity item with both edible and inedible stimuli.

Second, we increased the delay to reinforcement for the edible condition and decreased the delay to reinforcement for the inedible condition to create a short delay to reinforcement inedible condition and a long delay to reinforcement edible condition. If choice of the higher quantity stimulus by capuchin monkeys in the edible condition was driven by increased motivation due to relatively short delay to reinforcement, increasing that delay in the edible condition and 
decreasing it in the inedible condition should result in a reversal of performance patterns, with animals selecting the higher quantity stimulus more frequently in the inedible than the edible condition.

\section{Method}

Subjects Subjects were the same squirrel and capuchin monkeys who participated in Experiment 1, with the exception of one capuchin monkey subject who left the facility and therefore did not participate in Experiment 2. Her data from Experiment 1 were not included in analyses for this experiment.

\section{Conditions}

Delay to reinforcement Animals received a short delay to reinforcement inedible condition (short-delay inedible) and a long delay to reinforcement edible condition (long-delay edible). Methods were similar to those used in the edible and inedible conditions in Experiment 1, with a few changes. In the long-delay edible condition, subjects chose between edible stimuli, but instead of removing the food from the plate and handing it to the subject immediately, the experimenter lifted the top plate, reached into the empty bottom container (retrieved nothing), then took the food reward off the top plate and handed it to the subject. The delay between choice and the monkey receiving the reward averaged $5.53 \mathrm{~s}$ compared to $2.42 \mathrm{~s}$ in the short-delay edible condition from Experiment 1. In the short-delay inedible condition, the subject chose between inedible stimuli. Rather than lifting off the top plate to reveal the food rewards as in Experiment 1, the experimenter held the reward items for both choices concealed in her hands during the trial and offered them to the subject immediately after a choice was indicated. The delay between choice and reward averaged $0.89 \mathrm{~s}$ compared to $5.33 \mathrm{~s}$ in the long-delay inedible condition from Experiment 1.

Preferred food reward Food preference testing. A food preference test was conducted to determine the preferred food for each subject. Two novel foods were tested in pairwise comparison tests against the grapes used in Experiment 1. Monkeys were presented with one piece of each of the two foods per trial and received whichever food item they selected. All possible pairs of the three food types were presented five times per 15 trial session. Once the monkey chose a food item on at least $80 \%$ of trials on which it appeared they moved on to the preferred food reward testing conditions using this food item as the reward. The resulting preferred food items were dried mangoes for four capuchin monkeys, cashews for two capuchin monkeys, and mealworms for all six squirrel monkeys.

Preferred food reward training and testing. Animals received a preferred reward edible and preferred reward inedible condition. Methods, including delays to reinforcement, were identical to those used in the edible and inedible conditions in Experiment 1, except that instead of grapes, the food items used as stimuli in the edible condition and as reinforcers in both conditions were the preferred food item for each monkey.

Training and testing As in Experiment 1, each condition contained a training phase and a testing phase. Training and testing phases presented the same quantity pairings and used the same completion criteria as in Experiment 1. All monkeys received the four conditions, long-delay edible, short-delay inedible, preferred reward inedible, and preferred reward edible, in that order.

Data analysis Data from the edible and inedible conditions in Experiment 1 were used as comparisons to determine the impact of delay to reward and reward type modifications on choice of the higher quantity stimulus. A linear mixed-effect model, with subject as a random factor, compared performance on the short-delay grape reward edible and longdelay grape reward inedible conditions from Experiment 1 with performance on the four conditions in Experiment 2, with condition order, ratio between the choice quantities, distance between the choice quantities, delay (short, long), reward (grape, preferred), stimulus type (edible, inedible), species (capuchin, squirrel monkeys), and the interaction between species, reward, and stimulus type as fixed factors.

\section{Results}

Training As in Experiment 1, there were no differences in the number of training sessions required to reach criterion between conditions or species (linear mixed-effect model), condition $\times$ species, contrasts of condition, all $p s>.15$; contrast of species, $t(60)=1.44, p=.155,95 \%$ CI $[-0.26,2.26]$; contrasts of species $\times$ condition interaction, all $p \mathrm{~s}>.20$.

Testing As in Experiment 1, the linear mixed-effect model revealed a signficant effect of ratio (slope $=0.08, S E=$ $0.03), F(1,2298)=6.42, p=.011,95 \%$ CI $[0.02,0.13]$, and distance $($ slope $=0.05, S E=0.01), F(1,2298)=20.11, p<$ $.001,95 \%$ CI $[0.03,0.06]$, on performance consistent with Weber's law and the distance effect, suggesting that the task manipulations did not impact quantity comparison per se. While the testing conditions were presented in the same order for all subjects, there was no significant effect of condition order on performance, $F(1,2306)=0.06, p=.81,95 \% \mathrm{CI}$ $[-0.01,0.02]$, indicating that differences in performance across conditions were due to task manipulations rather than to general improvement over time. There was no significant effect of delay to reinforcement on performance (slope $=$ $-0.001, S E=0.02), F(1,2298)=0.00, p=.98,95 \% \mathrm{CI}$ $[-0.04,0.04]$ (see Fig. 3, right). The lack of an effect of delay 


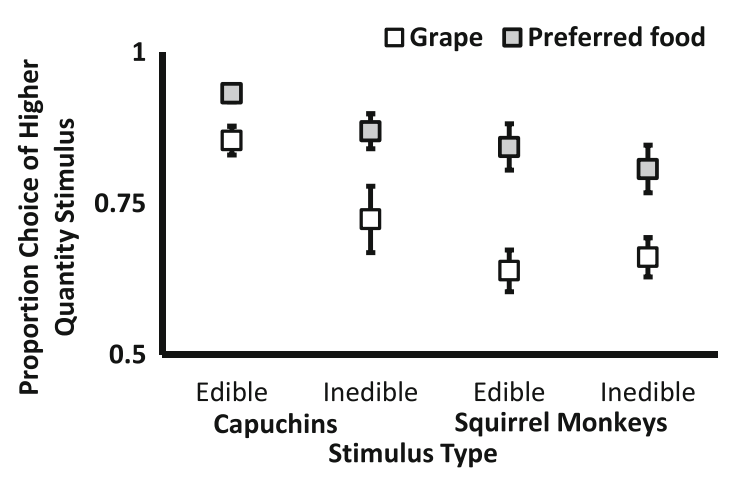

Short Delay

- Long Delay

Stimulus Type

Fig. 3 Proportion choice of higher quantity stimulus by capuchin and squirrel monkeys in the edible and inedible conditions across (left) grape and preferred reward types and (right) short and long delay conditions. Grape reward (open boxes), short-delay edible (light gray), and

to reinforcement indicates that increased choice of the higher quantity stimulus in the edible condition by capuchin monkeys in Experiment 1 was unlikely to be due to the short delay to reinforcment in that condition. It may be that delay to reinforcement was not a salient factor in assigning subjective reward value in this case.

The model additionally revealed a significant effect of species, such that capuchins continued to select the higher quantity stimulus more frequently than did squirrel monkeys (main effect of species), $F(1,10)=8.98, p=.013,95 \%$ CI $[-0.18$, $-0.46]$. Consistent with findings from capuchin monkeys in Experiment 1, the higher quantity item was chosen more frequently by New World monkeys presented with edible stimuli than inedible stimuli (main effect of stimulus type), $F(1,2298$ ) $=5.74, p=.017,95 \% \mathrm{CI}[-0.09,-0.01]$. Monkeys chose the higher quantity stimulus more frequently when reinforced with their preferred food item than when reinforced with the grapes used in Experiment 1 (main effect of reward type), $F(1$, $2305)=20.22, p<.001,95 \%$ CI $[-0.20,-0.08]$ (see Fig. 3, left). There were no significant interactions between species, stimulus type, or reward type (all $p \mathrm{~s}>.09$ ), indicating the positive effect of being rewarded with a preferred food was the same across capuchin and squirrel monkeys and across both edible and inedible stimuli. This indicates that for New World monkeys, increasing the subjective value of the reward by offering a preferred food increased the motivation to obtain higher quantities of reinforcers, regardless of the stimulus type.

\section{Discussion}

Consistent with findings that increasing motivation leads to improved cognitive performance in monkeys (Minamimoto et al., 2009; Schrier \& Harlow, 1956), results of the present study indicate that increasing the subjective value of the food reward by presenting a preferred food had a significant impact on choice behavior in the quantity discrimination task for both the capuchins and squirrel monkeys. Both species long-delay inedible (black) data are from Experiment 1, preferred reward (dark gray), long-delay edible (black), and short-delay inedible (light gray) data are from Experiment 2. Error bars indicate standard error of the mean

demonstrated increases in choice of the higher quantity stimulus when presented with a preferred food reward. This suggests that low levels of choice of the higher quantity stimulus in Experiment 1 by capuchins in the inedible condition and by squirrel monkeys in all conditions was driven by low subjective value of the reinforcer. This low subjective value was not due to increased delay to receive the reward, as manipulation of this delay did not impact choice behavior. The significant increase in choice of the higher quantity stimulus by squirrel monkeys with a preferred reward is an important one, as squirrel monkeys often show relatively poor performance on cognitive tasks (Tomasello \& Call, 1997). These results suggest that, rather than qualitative cognitive differences, squirrel monkeys may not be motivated in the same way by reward or experimental features as are other primate species. This may explain the variation in success between subjects and across studies on previous quantity-discrimination tasks by squirrel monkeys (Adachi et al., 2011; McKenzie et al., 2004; Thomas \& Chase, 1980; Thomas et al., 1980), as some task parameters may be more motivating or some subjects may be more motivated to attend to the task.

\section{General discussion}

In Experiment 1, New World monkeys showed two distinct choice patterns on a quantity-discrimination task across edible, inedible, and edible replaced conditions. Unlike Old World monkeys in Schmitt and Fischer's (2011) original study, capuchin monkeys choose the higher quantity stimulus most frequently in the edible condition. Squirrel monkeys performed equally poorly across all three conditions. While performance patterns by Old World monkeys may be best explained by difficultly representing food items as both choice stimuli and rewards (Schmitt \& Fischer, 2011), this explanation is not consistent with performance patterns by New World monkeys. Instead, performance patterns by both species may best be explained by differences in assignment of subjective 
reward value to the stimuli. Increasing the subjective value of the reward by offering a preferred food item in Experiment 2 increased choice of the higher quantity stimulus for both species and both stimulus types, indicating that motivation to obtain rewards plays an important role in performance on quantity discrimination tasks.

In all three conditions, the arrangement of the choice stimuli on the plates varied between trials, such that individual items could be located closer or further apart from each other. This prevented animals from using any set pattern to identify specific quantities. The marbles used as choice stimuli in the inedible condition were larger than the grape pieces used as choice stimuli in the edible and edible replaced conditions. These two features of the stimuli could have resulted in monkeys basing their choices on other magnitude features besides quantity - for example, the overall surface area of the items or the overall area of the plate covered by the items. The results of this study therefore cannot differentiate which specific mechanism monkeys were using to discriminate between quantities. However, New World monkeys did show abovechance performance, ratio, and distance effects on their choice behavior, indicating that they were attending to some consistent relative magnitude dimension of the stimuli. This compliments previous research in primates that show conformity to distance effects and Weber's lab on quantity discrimination tasks (Barnard et al., 2013; Cantlon \& Brannon, 2006; Evans et al., 2009; Hanus \& Call, 2007; Jordan \& Brannon, 2006; Merritt et al., 2011). These patterns are consistent with an approximate representation of quantity and indicate systematic comparison of the two choice quantities. This adds to the growing body of literature that suggests common magnitude processing mechanisms across species (Barnard et al., 2013; Evans et al., 2009; Gomez-Laplaza \& Gerlai, 2016; Hanus \& Call, 2007; Jordan \& Brannon, 2006; Kelly, 2016; Merritt et al., 2011; Roberts, 2005). That ratio and distance effects were not disturbed by stimulus type, reward type, or delay to reinforcement manipulations suggests that differences in absolute performance indicate differences at a stimulusprocessing level rather than differences in quantity comparison abilities.

While monkeys and apes generally show a preference for the larger quantity of food in natural choice procedures such as the one presented in the edible condition, variables such as food type, size, and intertrial interval can reverse this preference through changes in the subjective value of the reinforcer (Addessi et al., 2008; Beran, Evans, \& Harris, 2008a; Beran, Ratliff, \& Evans, 2009; Parrish, Evans, \& Beran, 2015; Sanchez-Amaro, Pereto, \& Call, 2016; Schmitt \& Fischer, 2011). Chimpanzees will select a smaller quantity of preferred food items over a larger quantity of nonpreferred food items (Beran et al., 2009; Silberberg, Widholm, Bresler, Fujita, \& Anderson, 1998) and show preferences for larger over smaller pieces of food, even when the small pieces add up to a larger overall amount (Parrish et al., 2015). These findings are consistent with results of the present experiments that suggest New World monkeys assign a higher subjective reward value to preferred food items. Delay to reinforcement may also influence subjective reward value (Beran et al., 2009; Sanchez-Amaro et al., 2016; Schubiger et al., 2016), although recent findings are mixed. Some studies find a significant impact of delay on choice behavior (Beran et al., 2009), others find no effect (Sanchez-Amaro et al., 2016), and still others find that effects vary based on task parameters (Schubiger et al., 2016). The results of Experiment 2 showed that even doubling the delay in the edible condition did not significantly influence choice behavior by New World monkeys. This indicates that delay to reinforcement was not an important factor in the subjective value of the reward in this task. The mixed findings around delay highlight that subjective reward value is likely influenced by a combination of context dependent factors including delay, quantity, and food type that vary across tasks (Parrish et al., 2015; Sanchez-Amaro et al., 2016).

That New World monkeys in this series of experiments consistently selected the higher quantity stimulus above chance supports previous findings that primates prefer items with higher subjective reward value when presented with a natural choice paradigm (Beran et al., 2009; Sanchez-Amaro et al., 2016; Schubiger et al., 2016). The present study adds to this literature by showing that presenting preferred food rewards resulted in an overall increase in performance, indicating that, in addition to influencing relative preferences, subjective reward value can significantly impact cognitive performance generally. These results indicate that differences in how species or individuals respond to methodological factors, such as stimulus and reward type, can result in significant differences in choice behavior, suggesting that any comparisons between species based on performance, even on identical tasks, need to be interpreted carefully. Specifically, these results highlight the importance of assuring adequate motivation in all species in comparative research, as differences in assignment of subjective value to rewards may result in performance differences that mask the cognitive abilities being tested. Elucidating the contributions of some of the many cognitive processes involved in producing the final behavioral output of interest is essential to valid characterization of phylogenetic distribution of cognitive processes.

Acknowledgments We thank the Bucknell animal care staff for caring for the animals and helping to separate animals for testing. We also thank Allie Schrock, Corinne Leard, Sarah Faid, and Meredith Lutz for assisting with data collection. This work was supported by a Griffith Faculty Fellowship to R.P.G. and a Bucknell PUR award to A.R.B. 
Author note Alison Billas is now at Penn Veterinary School, University of Pennsylvania, Philadelphia PA.

\section{References}

Adachi, I., Anderson, J. R., \& Fujita, K. (2011). Reverse-reward learning in squirrel monkeys (Saimiri sciureus): Retesting after 5 years, and assessment on qualitative transfer. Journal of Comparative Psychology, 125(1), 84-90. doi:10.1037/a0021041

Addessi, E., Crescimbene, L., \& Visalberghi, E. (2008). Food and token quantity discrimination in capuchin monkeys (Cebus apella). Animal Cognition, 11(2), 275-282. doi:10.1007/s10071-007-0111-6

Addessi, E., \& Rossi, S. (2011). Tokens improve capuchin performance in the reverse-reward contingency task. Proceedings of the Royal Society B-Biological Sciences, 278(1707), 849-854. doi:10.1098/ rspb.2010.1602

Agrillo, C., \& Bisazza, A. (2014). Spontaneous versus trained numerical abilities: A comparison between the two main tools to study numerical competence in non-human animals. Journal of Neuroscience Methods, 234, 82-91. doi:10.1016/j.jneumeth.2014.04.027

Agrillo, C., Dadda, M., \& Bisazza, A. (2007). Quantity discrimination in female mosquitofish. Animal Cognition, 10(1), 63-70. doi:10.1007/ s10071-006-0036-5

Agrillo, C., Dadda, M., Serena, G., \& Bisazza, A. (2008). Do fish count? Spontaneous discrimination of quantity in female mosquitofish. Animal Cognition, 11(3), 495-503. doi:10.1007/s10071-008-0140-9

Anderson, J. R., Awazu, S., \& Fujita, K. (2000). Can squirrel monkeys learn self control? A study using food array selection tests and reverse reward contingency. Journal of Experimental Psychology, 26, 87-97.

Anderson, J. R., Awazu, S., \& Fujita, K. (2004). Squirrel monkeys (Saimiri sciureus) choose smaller food arrays: Long-term retention, choice with nonpreferred food, and transposition. Journal of Comparative Psychology, 118(1), 58-64. doi:10.1037/0735-7036. 118.1.58

Anderson, U. S., Stoinski, T. S., Bloomsmith, M. A., \& Maple, T. L. (2007). Relative numerousness judgment and summation in young, middle-aged, and older adult Orangutans (Pongo pygmaeus abelii and Pongo pygmaeus pygmaeus). Journal of Comparative Psychology, 121(1), 1-11. doi:10.1037/0735-7036.121.1.1

Anderson, U. S., Stoinski, T. S., Bloomsmith, M. A., Marr, M. J., Smith, A. D., \& Maple, T. L. (2005). Relative numerousness judgment and summation in young and old Western lowland gorillas. Journal of Comparative Psychology, 119(3), 285-295. doi:10.1037/07357036.119.3.285

Baker, J. M., Shivik, J., \& Jordan, K. E. (2011). Tracking of food quantity by coyotes (Canis latrans). Behavioural Processes, 88(2), 72-75. doi:10.1016/j.beproc.2011.08.006

Barnard, A. M., Hughes, K. D., Gerhardt, R. R., DiVincenti, L., Bovee, J. M., \& Cantlon, J. F. (2013). Inherently analog quantity representations in olive baboons (Papio anubis). Frontiers in Psychology, 4, 11. doi:10.3389/fpsyg.2013.00253

Beran, M. J. (2001). Summation and numerousness judgments of sequentially presented sets of items by chimpanzees (Pan troglodytes). Journal of Comparative Psychology, 115(2), 181-191.

Beran, M. J. (2008). The evolutionary and developmental foundations of mathematics. PLOS Biology, 6(3), e19.

Beran, M. J., Evans, T. A., \& Harris, E. H. (2008a). Perception of food amounts by chimpanzees based on the number, size, contour length and visibility of items. Animal Behaviour, 75, 1793-1802. doi:10. 1016/j.anbehav.2007.10.035

Beran, M. J., Evans, T. A., Leighty, K. A., Harris, E. H., \& Rice, D. (2008b). Summation and quantity judgments of sequentially presented sets by capuchin monkeys (Cebus apella). American Journal of Primatology, 70(2), 191-194. doi:10.1002/ajp.20474

Beran, M. J., Ratliff, C. L., \& Evans, T. A. (2009). Natural choice in chimpanzees (Pan troglodytes): Perceptual and temporal effects on selective value. Learning and Motivation, 40(2), 186-196. doi:10. 1016/j.lmot.2008.11.002

Boisgontier, M. P., \& Cheval, B. (2016). The anova to mixed model transition. Neuroscience and Biobehavioral Reviews, 68, 1004 1005.

Bolker, B. M., Brooks, M. E., Clark, C. J., Geange, S. W., Poulsen, J. R., Stevens, M. H. H., \& White, J. S. S. (2009). Generalized linear mixed models: A practical guide for ecology and evolution. Trends in Ecology \& Evolution, 24(3), 127-135. doi:10.1016/j. tree.2008.10.008

Boysen, S. T., Berntson, G. G., Hannan, M. B., \& Cacioppo, J. T. (1996). Quantity-based interference and symbolic representations in chimpanzees (Pan troglodytes). Journal of Experimental PsychologyAnimal Behavior Processes, 22(1), 76-86.

Brucks, D., Soliani, M., Range, F., \& Marshall-Pescini, S. (2017). Reward type and behavioural patterns predict dogs' success in a delay of gratification paradigm. Scientific Reports, 7, 10. doi:10. $1038 /$ srep42459

Cantlon, J. F. (2012). Math, monkeys, and the developing brain. Proceedings of the National Academy of Sciences of the United States of America, 109, 10725-10732. doi:10.1073/pnas. 1201893109

Cantlon, J. F., \& Brannon, E. M. (2006). Shared system for ordering small and large numbers in monkeys and humans. Psychological Science, 17(5), 401-406.

Evans, T. A., Beran, M. J., Harris, E. H., \& Rice, D. (2009). Quantity judgments of sequentially presented food items by capuchin monkeys (Cebus apella). Animal Cognition, 12, 97-105.

Evans, T. A., Beran, M. J., Paglieri, F., \& Addessi, E. (2012). Delaying gratification for food and tokens in capuchin monkeys (Cebus apella) and chimpanzees (Pan troglodytes): When quantity is salient, symbolic stimuli do not improve performance. Animal Cognition, 15(4), 539-548. doi:10.1007/s10071-012-0482-1

Gomez-Laplaza, L. M., \& Gerlai, R. (2016). Discrimination of large quantities: Weber's law and short-term memory in angelfish, Pterophyllum scalare. Animal Behaviour, 112, 29-37. doi:10.1016/ j.anbehav.2015.10.022

Gossette, R. L., \& Feldman, J. (1968). An examination of the sensitivity of successive discrimination reversal (SDR) measures to differences in motivation level with squirrel monkeys. Psychonomic Science, 11(5), 157-158.

Gueorguieva, R., \& Krystal, J. H. (2004). Move over ANOVA. Archives of General Psychiatry, 61, 310-317.

Hanus, D., \& Call, J. (2007). Discrete quantity judgments in the great apes (Pan paniscus, Pan troglodytes, Gorilla gorilla, Pongo pygmaeus): The effect of presenting whole sets versus item-by-item. Journal of Comparative Psychology, 121(3), 241-249. doi:10.1037/ 0735-7036.121.3.241

Jordan, K. E., \& Brannon, E. M. (2006). Weber's Law influences numerical representations in rhesus macaques (Macaca mulatta). Animal Cognition, 9(3), 159-172. doi:10.1007/s10071-006-0017-8

Judge, P. G., \& Bruno, S. (2012). Transport of functionally appropriate tools by capuchin monkeys (Cebus apella). American Journal of Primatology, 74(3), 199-209. doi:10.1002/ajp.21987

Judge, P. G., Evans, D. W., Schroepfer, K. K., \& Gross, A. C. (2011). Perseveration on a reversal-learning task correlates with rates of selfdirected behavior in nonhuman primates. Behavioural Brain Research, 222(1), 57-65. doi:10.1016/j.bbr.2011.03.016

Judge, P. G., Evans, T. A., \& Vyas, D. K. (2005). Ordinal representation of numeric quantities by brown Capuchin monkeys (Cebus apella). Journal of Experimental Psychology-Animal Behavior Processes, 31(1), 79-94. 
Kelly, E. M. (2016). Counting on your friends: The role of social environment on quantity discrimination. Behavioural Processes, 128, 9 16. doi:10.1016/j.beproc.2016.03.019

Kilian, A., Yaman, S., von Fersen, L., \& Gunturkun, O. (2003). A bottlenose dolphin discriminates visual stimuli differing in numerosity. Learning \& Behavior, 31(2), 133-142.

Kurdziel, L. B., \& Judge, P. G. (2007). Picture recognition of food in brown capuchin monkeys (Cebus apella). American Journal of Primatology, 69, 100-100.

Kuznetsova, A., Brockhoff, P. B., \& Christensen, R. H. B. (2015). lmerTest: Tests in linear mixed effects models. https://cran.rproject.org/web/packages/lmerTest/lmerTest.pdf

Marsh, H. L., Vining, A. Q., Levendoski, E. K., \& Judge, P. G. (2015). Inference by exclusion in lion-tailed macaques (Macaca silenus), a hamadryas baboon (Papio hamadryas), capuchins (Sapajus apella), and squirrel monkeys (Saimiri sciureus). Journal of Comparative Psychology, 129(3), 256-267. doi:10.1037/a0039316

Mazur, J. E. (2001). Hyperbolic value addition and general models of animal choice. Psychological Review, 108(1), 96-112. doi:10. 1037/0033-295x.108.1.96

McKenzie, T., Cherman, T., Bird, L. R., Naqshbandi, M., \& Roberts, W. A. (2004). Can squirrel monkeys (Saimiri sciureus) plan for the future? Studies of temporal myopia in food choice. Learning \& Behavior, 32(4), 377-390.

Merritt, D. J., Maclean, E. L., Crawford, J. C., \& Brannon, E. M. (2011). Numerical rule-learning in ring-tailed lemurs (Lemur catta). Frontiers in Psychology, 2(23). doi:10.3389/fpsyg.2011.00023

Minamimoto, T., La Camera, G., \& Richmond, B. J. (2009). Measuring and modeling the interaction among reward size, delay to reward, and satiation level on motivation in monkeys. Journal of Neurophysiology, 101(1), 437-447. doi:10.1152/jn.90959.2008

Olthof, A., Iden, C. M., \& Roberts, W. A. (1997). Judgments of ordinality and summation of number symbols by squirrel monkeys (Saimiri sciureus). Journal of Experimental Psychology-Animal Behavior Processes, 23(3), 325-339. doi:10.1037/0097-7403.23.3.325

Panteleeva, S., Reznikova, Z., \& Vygonyailova, O. (2013). Quantity judgments in the context of risk/reward decision making in striped field mice: First "count," then hunt. Frontiers in Psychology, 4, 8. doi:10.3389/fpsyg.2013.00053

Parrish, A. E., Evans, T. A., \& Beran, M. J. (2015). Defining value through quantity and quality: Chimpanzees (Pan troglodytes) undervalue food quantities when items are broken. Behavioural Processes, 111, 118-126. doi:10.1016/j.beproc.2014.11.004

R Core Team. (2014). R: A language and environment for statistical computing. Vienna: R Foundaton for Statistical Computing. Retrieved from http://www.R-project.org/

Roberts, W. A. (2005). How do pigeons represent numbers? Studies of number scale bisection. Behavioral Processes, 69, 33-43.

Sanchez-Amaro, A., Pereto, M., \& Call, J. (2016). Differences in between-reinforcer value modulate the selective-value effect in great apes (Pan troglodytes, P. paniscus, Gorilla gorilla, Pongo abelii). Journal of Comparative Psychology, 130(1), 1-12. doi:10.1037 com0000014

Schmitt, V., \& Fischer, J. (2011). Representational format determines numerical competence in monkeys. Nature Communications, 2(257). doi:10.1038/ncomms 1262

Schrier, A. M., \& Harlow, H. F. (1956). Effect of amount of incentive on discrimination learning by monkeys. Journal of comparative physiological psychology, 49(2), 117-122.

Schubiger, M. N., Kissling, A., \& Burkart, J. M. (2016). How task format affects cognitive performance: A memory test with two species of New World monkeys. Animal Behaviour, 121, 33-39. doi:10.1016/j. anbehav.2016.08.005

Silberberg, A., Widholm, J. J., Bresler, D., Fujita, K., \& Anderson, J. R. (1998). Natural choice in nonhuman primates. Journal of Experimental Psychology-Animal Behavior Processes, 24(2), 215-228.

Terrell, D. F., \& Thomas, R. K. (1990). Number-related discrimination and summation by squirrel monkeys (Saimiri sciureus sciureus and $S$. boliviensis boliviensis) on the basis of the number of sides of polygons. Journal of Comparative Psychology, 104(3), 238-247. doi:10.1037/0735-7036.104.3.238

Thomas, R. K., \& Chase, L. (1980). Relative numerousness judgements by squirrel monkeys. Bulletin of the Psychonomic Society, 16(2), $79-82$.

Thomas, R. K., Fowlkes, D., \& Vickery, J. D. (1980). Conceptual numerousness judgments by squirrel monkeys. American Journal of Psychology, 93(2), 247-257.

Tomasello, M., \& Call, J. (1997). Primate cognition. New York: Oxford University Press.

Truppa, V., Carducci, P., Trapanese, C., \& Hanus, D. (2015). Does presentation format influence visual size discrimination in tufted capuchin monkeys (Sapajus spp.)? PLOS ONE, 10(4), 18. doi:10.1371/ journal.pone. 0126001

Utrata, E., Viranyi, Z., \& Range, F. (2012). Quantity discrimination in wolves (Canis lupus). Frontiers in Psychology, 3, 9. doi:10.3389/ fpsyg.2012.00505

Veling, H., \& Bijleveld, E. (2015). When performance and risk taking are related: Working for rewards is related to risk taking when the value of rewards is presented briefly. Brain and Cognition, 101, 44-50. doi:10.1016/j.bandc.2015.11.001

Vlamings, P. H. J. M., Uher, J., \& Call, J. (2006). How the great apes (Pan troglodytes, Pongo pygmaeus, Pan paniscus, and Gorilla gorilla) perform on the reversed contingency task: The effects of food quantity and food visability. Journal of Experimental Psychology: Animal Behavior Processes, 32, 60-70.

Zander, S. L., \& Judge, P. G. (2015). Brown capuchin monkeys (Sapajus apella) plan their movements on a grasping task. Journal of Comparative Psychology, 129(2), 181-188. doi:10.1037/a0038850 